\title{
Fluorescent Hyperbranched Polyamine with $s$-Triazine: Synthesis, Characterization and Properties Evaluation
}

\author{
By Sibdas S. MAHAPATRA and Niranjan KARAK*
}

\begin{abstract}
A hyperbranched polyamine with $s$-triazine unit based on 2,4,6-trichloro-s-triazine and 4,4'-oxydianiline has been synthesized by using $\mathrm{A}_{2}+\mathrm{B}_{3}$ approach. This polyamine was characterized by FT-IR, UV-visible, ${ }^{1} \mathrm{H}$ NMR, ${ }^{13} \mathrm{C}$ NMR spectroscopic, elemental analysis, measurement of solution viscosity, X-ray diffraction and solubility studies. The terminal amino groups of the polymer were dye end capped by methyl red moiety. The hyperbranched polyamine found to display strong fluorescence from $350 \mathrm{~nm}$ to $650 \mathrm{~nm}$ in DMSO solution. The fluorescence intensity increased significantly by end capping with methyl red. The hyperbranched polyamine also exhibit $\mathrm{pH}$ sensitive fluorescence and the intensity increases with the increase of $\mathrm{pH}$ of the medium. The $\mathrm{Cu}^{2+}$ ion quenched the fluorescence of the polymer solution noticeably. The polymer solution also shows the increase of fluorescence intensity with the decrease in concentration of polymer. The hyperbranched polyamine was soluble only highly polar solvents and exhibits low viscosity $\left(\eta_{\text {inh }}=0.15 \mathrm{dL} / \mathrm{g}\right)$. The thermal analyses indicate that the polymer is thermostable upto $300^{\circ} \mathrm{C}$ with glass transition $\left(T_{\mathrm{g}}\right)$ temperature $238^{\circ} \mathrm{C}$, while by end capping $T_{\mathrm{g}}$ further increased to $271^{\circ} \mathrm{C}$. The polymer also exhibits self-extinguishing characteristic as found by limiting oxygen index (LOI) value measurement.

KEY WORDS: Hyperbranched Polyamine / End Capped / $\mathrm{A}_{2}+\mathrm{B}_{3}$ Approach / s-Triazine / Fluorescence / Thermal Property /
\end{abstract}

Over the last two decades hyperbranched polymers have attracted considerable attention ${ }^{1-4}$ for their unusual properties like low viscosity, high solubility, unique three-dimensional structure and globular shape etc. in different field of applications. ${ }^{5-7}$ Among these, fluorescence is one of the most important applications as it has been applied in many areas especially in chemical and biological sciences. ${ }^{8,9}$ Further conjugate structures with electron deficient unit such as nitrogen containing heterocyclic moiety are very interesting for this study. ${ }^{10,11}$ The design of a polymer with heterocyclic moiety like $s$-triazine unit in the structure has profound effects on the physico-chemical and thermal properties as well as on its processability. ${ }^{12,13}$ The incorporation of rigid triazine unit in the hyperbranched polymeric structure is largely due to the case of chemo- and thermo-selective substitution of the chlorine atoms with different nucleophiles to generate a variety of structures. The three chlorine atoms of 2,4,6-trichloro-striazine (cyanuric chloride) are substituted by the amine nucleophiles consecutively at $0-5{ }^{\circ} \mathrm{C}, 40-45^{\circ} \mathrm{C}$ and $80-90^{\circ} \mathrm{C}$ in a single pot reaction, while the reaction is carried out with some precaution. ${ }^{14,15}$ There are a large number of reports on the fluorescence studies of hyperbranched polymers with different structures. ${ }^{16-19}$ However, the studies on fluorescence of aromatic hyperbranched polyamine with $s$-triazine unit are not known to our knowledge. Again, this type of hyperbranched polymers exhibits many interesting properties like high thermostability, self-extinguishing characteristics, easy of processing etc. Further, three-dimensional structures of hyperbranched polymers help to reduce the aggregation of the conjugated polymer chains and hence improve the luminescent efficiency. Thus in the present investigation $s$-triazine containing hyperbranched polyamine is used for the first time to study the fluorescence behavior in solution state. This $1,3,5$ triazine has also two important properties that incline to use this in this study. These are (i) its ionization potential value of $11.67 \mathrm{eV}$, i.e., it is more electron deficient than pyridine $(9.73 \mathrm{eV})$, pyridazine $(10.61 \mathrm{eV})$, pyrimidine $(10.41 \mathrm{eV})$ and pyrazine $(10.2 \mathrm{eV})$ and (ii) unlike benzene ring connecting three aromatic ring at 2, 4 and 6 positions of 1,3,5-triazine will not create the steric interactions of the ortho hydrogens. $^{20}$

Further end capping with dye molecules or complexation with metal ions influence the fluorescence intensity of hyperbranched polymer by changing the quenching effects of the intra or inter molecular interactions of the different functional groups. For example, fluorescent chemosensors for selective detection of transition metal ions especially $\mathrm{Cu}^{2+}$ ion are rather rare, ${ }^{21,22}$ although it is a very important metal in human body and plays an important role in various physiological processes. So the effect of end capping and metal ion complexation are very important. ${ }^{16-18}$ Similarly the change of $\mathrm{pH}$ also affects the fluorescence intensity of the hyperbranched polymers, ${ }^{15-17,19}$ hence need to investigate for such study.

Authors, therefore, wish to report the synthesis, characterization and properties evaluation of a fluorescent hyperbranched aromatic polyamine with $s$-triazine unit. The effect of dye end capping is also investigated. The fluorescence of the polymer has also been studied with the variation of concentration, $\mathrm{pH}$ of the medium and in the presence of $\mathrm{Cu}^{2+}$ ion. 


\section{EXPERIMENTAL}

\section{Materials}

$N, N^{\prime}$-Dimethyl acetamide (DMAc, Merck, India) and dimethyl sulphoxide (DMSO, Merck, India) were used after purification by vacuum distillation over calcium oxide and anhydrous calcium sulfate respectively. 2,4,6-Trichloro-1,3,5triazine (CYC), diisopropyl ethylamine (DIPEA, Merck, Germany), methyl red, cupric acetate and thionyl chloride (Merck, India) were used as received. 4,4'-oxydianiline (ODA, Aldrich Chemie, Germany) were used after purification from aqueous ethanol.

\section{Synthesis of Hyperbranched Polyamine (HBPA)}

$0.01 \mathrm{~mol}$ of $\mathrm{CYC}$ in $25 \mathrm{~mL}$ DMAc was added very slowly for about $1 \mathrm{~h}$ at $0-5{ }^{\circ} \mathrm{C}$ in $24 \mathrm{~mL}$ solution of $0.015 \mathrm{~mol}$ of ODA and $0.01 \mathrm{~mol}$ of DIPEA (diisopropyl ethylamine) in DMAc. After $1 \mathrm{~h}$, temperature increased to $40-45^{\circ} \mathrm{C}$ and $0.01 \mathrm{~mol}$ of DIPEA was added into the reaction mixture. Then the temperature increased to $85-90{ }^{\circ} \mathrm{C}$ after $2 \mathrm{~h}$, and $0.01 \mathrm{~mol}$ of DIPEA was added again. The mixture was cooled after $5 \mathrm{~h}$ and poured slowly in ice cold water. The precipitate was collected and washed several times with methanol and acetone to remove the unreacted reagents. The product was dried under vacuum at temperature $45-50{ }^{\circ} \mathrm{C}$ for $3 \mathrm{~d}$ to obtain dry powder of the polymer.

\section{Preparation of End Capped Hyperbranched Polyamine (HBPE)}

The end capping of hyperbranched polyamine was prepared by the following two step process. At first, $1.8 \mathrm{~g}(6.7 \mathrm{mmol})$ of methyl red and $5 \mathrm{~mL}$ of thionyl chloride were refluxed together for $6 \mathrm{~h}$. Then the excess thionyl chloride was removed under vacuum and the deep brown solid powered of chloride derivative of methyl red was obtained.

In the next step, $1 \mathrm{~g}\left(35 \times 10^{-4} \mathrm{~mol}\right)$ methyl red chloride in $10 \mathrm{~mL}$ DMAc and $4.53 \mathrm{~g}$ of DIPEA were added dropwise in $1 \mathrm{~g}$ $\left(1.75 \times 10^{-4} \mathrm{~mol}\right)$ of DMAc solution of HBPA with constant stirring. The mixture was stirred for $6 \mathrm{~h}$ at $80{ }^{\circ} \mathrm{C}$, cooled to room temperature and then poured into ice cold water. The precipitate was filtered, washed with methanol and dried under vacuum at $45^{\circ} \mathrm{C}$ for $3 \mathrm{~d}$.

$\left[{ }^{1} \mathrm{H}\right.$ NMR, DMSO- $d_{6}(\delta, \mathrm{ppm}): 10.81,9.12$, and $9.22(-\mathrm{NH})$, 8.01 (-CONH), 6.68-7.61 (aromatic), 2.85 (aliphatic $-\mathrm{CH}_{3}$ ). FT-IR (KBr, cm ${ }^{-1}$ ) 3350-3370, 1636, 1580-1620.]

\section{Measurements}

FT-IR spectra for the compounds were recorded in a Nicolet (Impact 410, Madison, USA) FT-IR spectrophotometer by using $\mathrm{KBr}$ pellets. ${ }^{1} \mathrm{H} \mathrm{NMR}$ and ${ }^{13} \mathrm{C}$ NMR spectra of the polymer were recorded with $400 \mathrm{MHz}$ NMR spectrometer (Varian, USA) by using $d_{6}$-DMSO as the solvent and TMS as the internal standard. The molecular weights of hyperbranched was determined by GPC analysis (Water, USA, Model 515) using DMSO as the solvent. The X-ray diffraction study was made on the powder sample at room temperature $\left(\mathrm{ca} .27^{\circ} \mathrm{C}\right)$ on a Rigaku X-ray diffractometer (Miniflex, UK). The scanning rate used was $5.0^{\circ} \mathrm{min}^{-1}$ over the range of $2 \theta=10-80^{\circ}$ for the above study. UV spectra of samples were recorded in a Hitachi (U-2001, Tokyo, Japan) UV spectrophotometer by using $0.001 \%$ solution in DMSO at room temperature (ca. $27^{\circ} \mathrm{C}$ ). The inherent viscosity of the polymer was measured using $0.5 \%(\mathrm{w} / \mathrm{v})$ solution of the polymer in DMAc at $27 \pm 0.1^{\circ} \mathrm{C}$ by an Ubbelohde suspended level viscometer. Thermogravimetric (TG) analysis and differential scanning calorimetry (DSC) were carried out in Shimazdu TG 50 and DSC 60 thermal analyzers respectively using the nitrogen flow rate of $30 \mathrm{~mL} /$ min and at the heat rate of $10^{\circ} \mathrm{C} / \mathrm{min}$. The fluorescence spectra of the compounds were recorded in Perkin Elmer (LS55) spectrophotometer. The density of the polymer was determined by pycnometer in dry toluene at room temperature $\left(\mathrm{ca} .27^{\circ} \mathrm{C}\right)$ by the conventional liquid displacement method. The chlorine content of the polymer was determined by the standard Schoniger oxygen combustion method. The elemental analysis (CHN) was carried out in a Perkin Elmer elemental analyzer (model no. 2400 series II).

\section{RESULTS AND DISCUSSION}

\section{Synthesis and Characterization of Hyperbranched Poly- amine}

The $s$-triazine based hyperbranched aromatic polyamine of 4,4'-oxydianiline (ODA) as an $\mathrm{A}_{2}$ monomer and 2,4,6-trichloro 1,3,5-triazine (CYC) as a $\mathrm{B}_{3}$ monomer was synthesized by an $\mathrm{A}_{2}+\mathrm{B}_{3}$ approach (Scheme 1). The substitution reaction of CYC by the aromatic diamine is carried out at three different

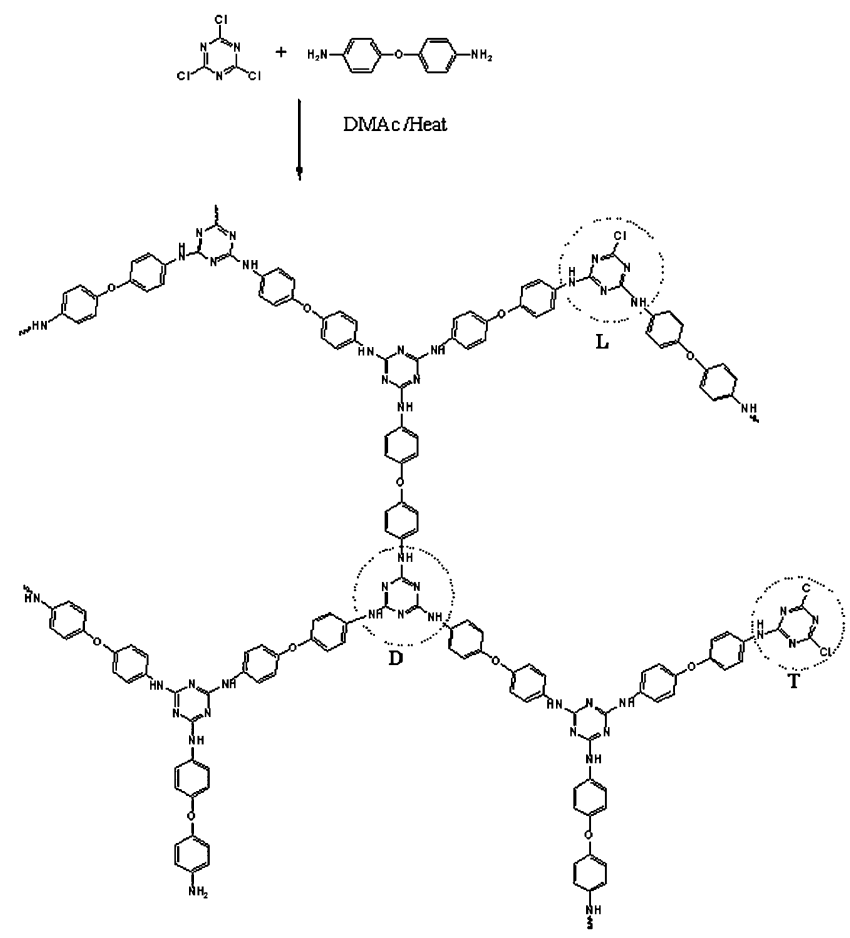

Scheme 1. Synthesis of hyperbranched polyamine. 
(a)

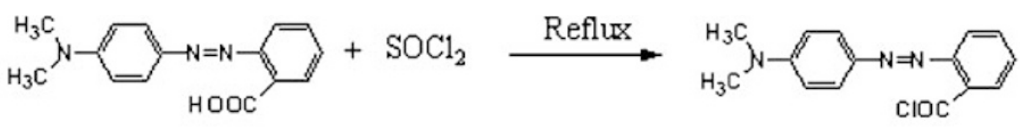

(b)

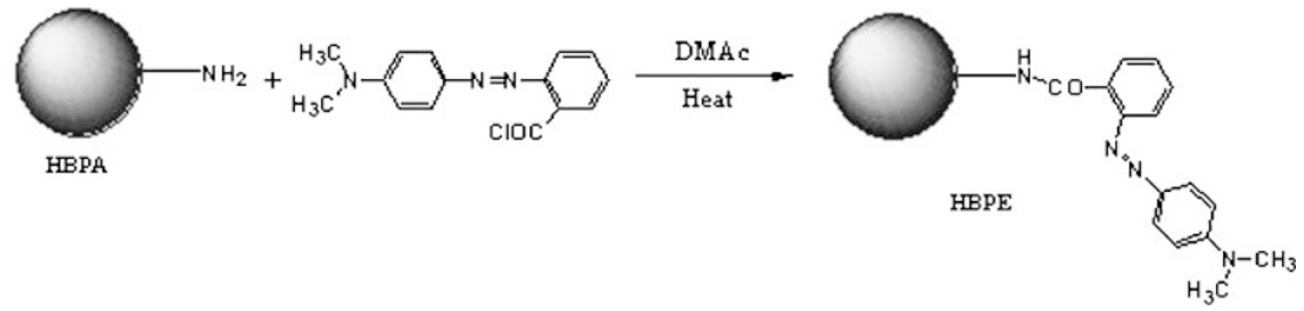

Scheme 2. End capping reaction of methyl red with hyperbranched polyamine.

temperatures as the reactivity of three chlorine atoms in $\mathrm{CYC}$ is highly thermoselective. ${ }^{14}$ Gelation is a common problem for this type of polymerization reaction. It was avoided by using appropriate ratio (3:2 mole ratio of $\mathrm{A}_{2}$ and $\mathrm{B}_{3}$ monomers), concentration $(<10 \%, \mathrm{w} / \mathrm{v})$ and slow addition of the monomers. These reaction conditions not only offered the product without gel but also a good yield (75\%). The end capping with methyl red was performed through the reaction of chloride derivative of the methyl red with the active surface primary amine group of the hyperbranched polyamine as shown in Scheme 2. As the acid chloride is more reactive to primary amine than the corresponding acid, so the chloride derivative was prepared first. The amorphous nature of the polymer is confirmed by XRD study. The polymer has moderate density $(1.18 \mathrm{~g} / \mathrm{cc})$ and light brown color with inherent viscosity $(0.15 \mathrm{dL} / \mathrm{g})$. This low viscosity may not only be due to low molecular weight of the polymer but also due to globular type structure. This is also confirmed by the determination of molecular weight (weight average molecular weight, $M_{\mathrm{w}}=$ $5700 \mathrm{~g} / \mathrm{mole}$ and polydispersity index 1.3) of the polymer. From the UV-visible spectra, the $\lambda_{\max }$ values of the polymers, HBPA and HBPE and methyl red dye in DMAc solution were observed at $280 \mathrm{~nm}$ and $315 \mathrm{~nm}, 416 \mathrm{~nm}$ respectively, which indicate the presence of aromatic moiety with conjugation and characteristic $\pi-\pi^{*}$ electronic transition in the aromatic 1,3,5triazine compounds. ${ }^{23}$ So a red shift of about $35 \mathrm{~nm}$ is observed for end capping with dye molecule. But from the spectra it is difficult to correlate the absorbance values, as their $\lambda_{\max }$ values are different. However, the introduction efficiency of dye molecule in the polymer can be calculated from the elemental analysis data of the polymers by the following procedure. The number average molecular weight of HBPA is $4,384 \mathrm{~g} / \mathrm{mole}$ (obtained from GPC data) and hence molecular weight of the repeat unit (taking two unit of triazine with three unit of diamine, as the reaction ratio) is $534 \mathrm{~g} /$ mole, so the number of such repeat unit is approximately 8 . That is approximately 16 triazine units and 25 diamine units are present in the structure. Now by utilizing the $\mathrm{DB}=0.58$ (obtained from ${ }^{1} \mathrm{H}$ NMR as described below), the number of free amino groups is
Table I. Elemental analysis data $(\mathrm{C} / \mathrm{H}$ and $\mathrm{N} / \mathrm{H}$ ratio $)$ of the hyperbranched unmodified and end capped polyamines

\begin{tabular}{clcl}
\hline \multirow{2}{*}{ polymer } & & $\begin{array}{c}\text { ratio of element } \\
\mathrm{C} / \mathrm{H}\end{array}$ & $\mathrm{C} / \mathrm{N}$ \\
\hline Pure HBPA & Calculated $^{\mathrm{a}}$ & 9.6 & 1.71 \\
& Found $_{\text {End capped }}$ & 9.15 & 3.91 \\
polymer & Calculated $^{\mathrm{b} 1}$ & 10.6 & 1.8 \\
& Calculated $^{\mathrm{b} 2}$ & 12.25 & 1.87 \\
& Calculated $^{\mathrm{b} 3}$ & 13.44 & 1.94 \\
& Calculated $^{\mathrm{b} 6}$ & 18.8 & 2.13 \\
& Calculated $^{\mathrm{b} 9}$ & 26.51 & 2.28 \\
& Found & 19.9 & 2.1 \\
\hline
\end{tabular}

${ }^{a}$ Calculated using ideal dendritic structure with no chlorine atom. bn Theoretically calculated taking different degree of dye substitution, $\mathrm{n}=1,2,3,6$ and 9 indicate number of dye molecules per molecule of end capped hyperbranched polyamine.

calculated in the structure and found to be approximately 10 . Now, the $\mathrm{C} / \mathrm{H}$ and $\mathrm{C} / \mathrm{N}$ ratios of the polymers are calculated taking ideal dendritic structures and experimentally found same values are tabulated in Table I. From this table it is found that the ratios vary continuously with different degree of substitution. So the plot of number of dye molecules per molecule of end capped hyperbranched polymer resulted almost straight line both the cases with varying slope. By fitting the experimentally observed ratio of $\mathrm{C} / \mathrm{H}$ and $\mathrm{C} / \mathrm{N}$ of the end capped polymer in this plot in origin software, the number of dye molecules is found to be 6.4-6.8 (6.4 from $\mathrm{C} / \mathrm{H}$ and 6.8 from $\mathrm{C} / \mathrm{N}$ ratio). Thus the introduction efficiency is approximately $66 \%$.

The important linkages in FT-IR spectrum like aromatic $\mathrm{N}-\mathrm{H}$ (primary or secondary) at $3255-3445 \mathrm{~cm}^{-1}, 1593-1621$ $\mathrm{cm}^{-1}$ and $1497 \mathrm{~cm}^{-1.24}$ supported the occurrence of nucleophilic displacement reaction. The marginal shift of these absorption values may be due to the presence of H-bonding. ${ }^{24}$ The other linkages like $\mathrm{C}=\mathrm{N}$ bond of the triazine moiety at $1412 \mathrm{~cm}^{-1}$ and aromatic $\mathrm{C}-\mathrm{Cl}$ bond at $1010 \mathrm{~cm}^{-1}$ were also found in the spectrum. ${ }^{24}$ The presence of $\mathrm{C}-\mathrm{Cl}$ bond indicates the displacement reaction was not completed. This was 


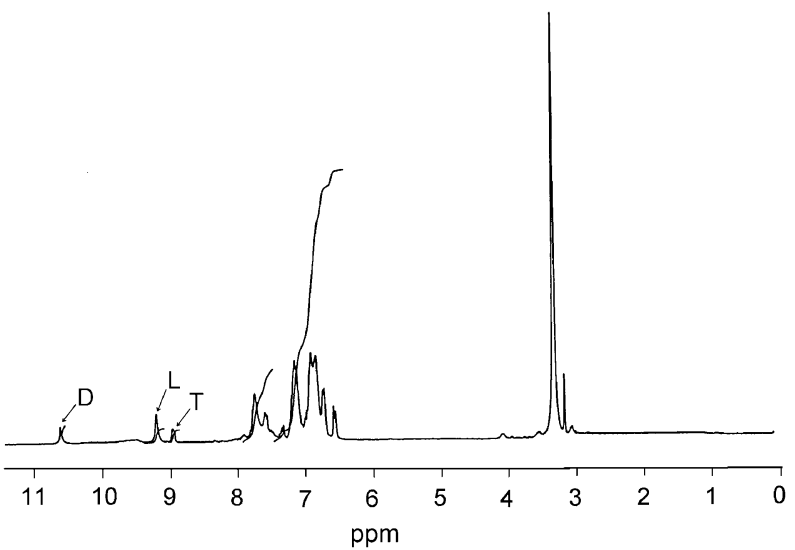

Figure 1. ${ }^{1} \mathrm{H}$ NMR spectrum of hyperbranched polyamine.

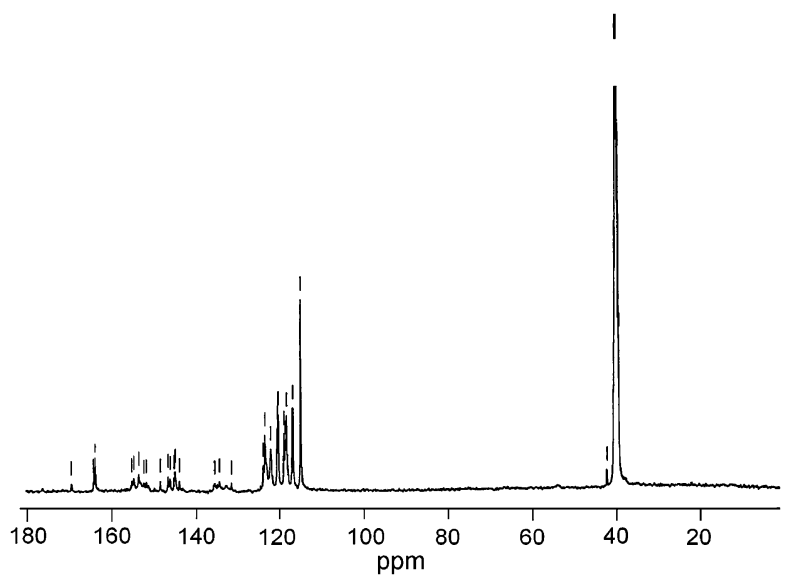

Figure 2. ${ }^{13} \mathrm{C}$ NMR spectrum of hyperbranched polyamine.

confirmed by quantitative measurement of chlorine by Schoniger oxygen combustion method (Table I). The ${ }^{1} \mathrm{H}$ NMR spectrum (Figure 1) of the polymer further indicates the presence of most important peaks for aromatic N-H protons. The dendritic (triazine with no chlorine atom), linear (triazine with one unsubstituted chlorine atom) and terminal units (triazine with two unsubstituted chlorine atoms) in the structure of the polymer are confirmed by the peaks at $\delta=10.66 \mathrm{ppm}$, $\delta=9.20 \mathrm{ppm}$ and $\delta=9.08 \mathrm{ppm}$, which are due to above three different $>\mathrm{N}-\mathrm{H}$ protons. ${ }^{25}$ The other protons like $-\mathrm{NH}_{2}$ at $\delta=3.21 \mathrm{ppm}$ and aromatic moieties at $\delta=6.50-7.76 \mathrm{ppm}$ as multiplate are also observed in the ${ }^{1} \mathrm{H}$ NMR spectrum. ${ }^{24}$ The ${ }^{13} \mathrm{C}$ NMR spectrum (Figure 2) indicates the presence of peak at $\delta=169.57 \mathrm{ppm}$ is due to unsubstitute chlorine atom in triazine ring, $\delta=163.98 \mathrm{ppm}$ (due to the carbon atoms present in the dendritic triazine units), ${ }^{26}$ peaks at $\delta=152.44-143.88 \mathrm{ppm}$ (due to the carbons directly attached with oxy groups), peaks at $\delta=135.37-131.37 \mathrm{ppm}$ (due to the carbon atoms directly attached with $-\mathrm{NH}$ groups) and peaks at $\delta=123.73-114.89$ ppm (for the carbon atoms at ortho and meta to oxy group). ${ }^{24}$

\section{Degree of Branching}

The degree of branching (DB) of the polymer is determined

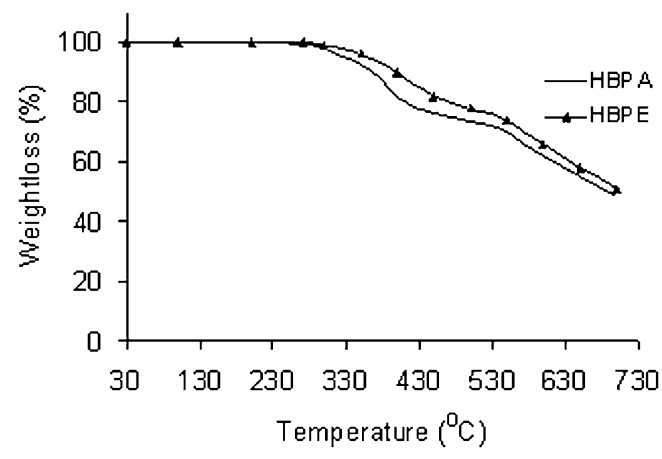

Figure 3. TG thermograms for HBPA and HBPE.

by using Frechet's equation as follow ${ }^{27}$ using ${ }^{1} \mathrm{H}$ NMR spectrum.

$$
\mathrm{DB}=(\mathrm{D}+\mathrm{T}) /(\mathrm{D}+\mathrm{T}+\mathrm{L})
$$

where $\mathrm{D}, \mathrm{T}$, and $\mathrm{L}$ refer to the number of dendritic, terminal and linear units in the structure of the polymer respectively. Experimentally, DB was determined from ${ }^{1} \mathrm{H}$ NMR spectroscopy by comparing the integration of the peaks for the respective units in the polymer (Figure 1). The value of DB for the polymer is found to be 0.58 . This value indicates that the polymer exhibits near to highly branched structure rather than more linear (DB close to zero for linear, 0.5 for hyperbranched and 1.0 for dendrimer) structure. ${ }^{28}$

\section{Properties of Polymer}

From the solubility studies it has been found that the polymer is soluble only in highly polar solvents such as DMAc, DMF, DMSO, NMP, etc., partially soluble in acetone, THF, etc. but insoluble in most of the other solvents like hydrocarbon solvents, water, chlorinated common organic solvents, etc. This may be due to the presence of polar $-\mathrm{NH}$ groups, rigid triazine units and aromatic moieties in the polymer structures. This higher solubility not only supported the hyperbranched formation but also confirmed that the polymer is not a crosslinked gel product. Further it has been found that the solubility further enhanced by addition of $\mathrm{HCl}$, which may be due to reduction of inter molecular attraction among the chain molecules.

The thermogravimatric analysis of HBPA and HBPE (Figure 3) showed that the initial degradation temperature, amount degraded at $400^{\circ} \mathrm{C}$, weight residue at $700^{\circ} \mathrm{C}$ and overall thermostability of HBPE is better than HBPA, although both the polymers exhibit good thermostability. The initial decomposition temperature of HBPA is $300^{\circ} \mathrm{C}$ whereas HBPE has $315^{\circ} \mathrm{C}$. The overall high thermostability of the polymers is due to the presence of thermosatable rigid triazine and aromatic moieties in the structures. ${ }^{29,30}$ However, the higher value for HBPE is due incorporation of two more aromatic moieties along with a thermostable. $\mathrm{N}=\mathrm{N}$ linkage in the structure of the end capped polymer. This is also reflected in the weight loss values at $400{ }^{\circ} \mathrm{C}$ and the weight residue at $700{ }^{\circ} \mathrm{C}(20 \%$ and $48 \%$ for HBPA and $15 \%$ and $52 \%$ for HBPE respectively). The 


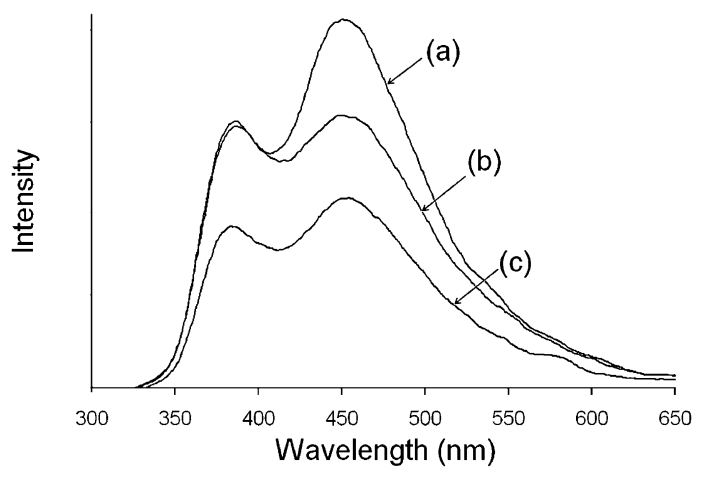

Figure 4. Fluorescence spectra of (a) HBPE (b) HBPA (c) HBPA-Cu complex.

high carbon to hydrogen ratio and the presence of special elements like nitrogen make both the polymer self-extinguishing in nature. The good flame retardancy of the polymers is also supported by high LOI value (36 for HBPA and 38 for HBPE).

In DSC studies no melting or crystallization peak was observed, which indicates that this hyperbranched polymer is amorphous in nature, which supports the XRD result. The polymer has $T_{\mathrm{g}}$ of $238^{\circ} \mathrm{C}$ as obtained from DSC and this indicates the rigidity of the structure. ${ }^{31}$

\section{Fluorescence Study}

This hyperbranched polyamine containing all three different type of nitrogen linkages, viz., primary at terminal, secondary in main chain and tertiary in triazine unit. The fluorescence emissions of hyperbranched polymer $\left(0.01 \%, 1.6 \times 10^{-5}\right.$ $\mathrm{mol} / \mathrm{L}$ in DMSO), its end-capped derivative with methyl red dye $(0.01 \%)$ and complex with $\mathrm{Cu}^{2+}$ ion $(0.015 \%)$. were shown in Figure 4. All three samples were excited with a $285 \mathrm{~nm}$ wavelength of light and exhibit emission in the yellow to blue region from 350 to $650 \mathrm{~nm}$ with maxima at about $455 \mathrm{~nm}$ and a shoulder at about $390 \mathrm{~nm}$. From these results it has been observed (Figure 4) that end capping enhanced the intensity of fluorescence significantly. This may be due to the facts that end capping prominently render the quenching process of energy and/or electron transfer processes. ${ }^{16,17}$ These processes otherwise could have been occurred between electron donating primary and/or secondary amino groups and electron accepting aromatic and heterocyclic moieties present in the structure of the polymer (Scheme 1). Further the dye molecule also enhances the conjugation and hence increases intensity. The quantum yield was determined by using anthracene with quantum yield 0.27 in ethanol is as the standard compound. The quantum yields of unmodified and end capped polymers are found to be 0.08 and 0.089 respectively. The study of fluorescence of hyperbranched polyamine in the presence of metal ions is very interesting due to its high-branched structure and the presence of different co-ordination sites. In case of $\mathrm{Cu}^{2+}$ ion-polymer complex solution, the intensity decreases due to intracomplex quenching effect. Since the hyperbranched polyamine is a 'push pull' system, excitation leads to a

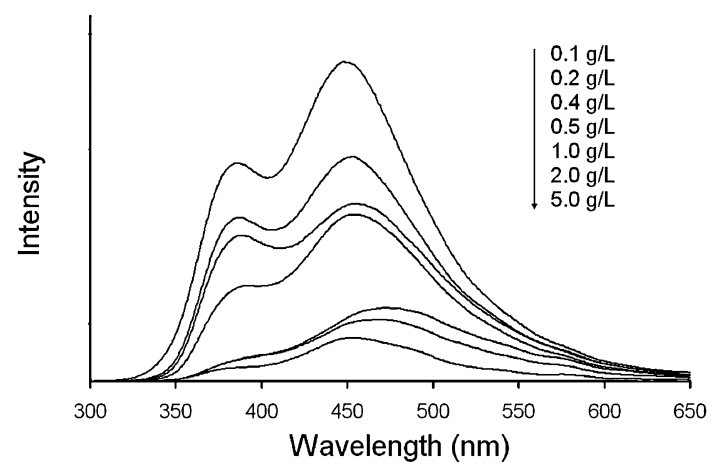

Figure 5. The fluorescence spectra of HBPA with variation of concentration.

significant redistribution of electron density so that a substantial dipole may be created. ${ }^{32}$ This attributed to energy transfer quenching between the complexing elements.

\section{Effect of Concentration}

The hyperbranched polyamine exhibit a general concentration 'self-quenching' effect with increasing concentration of polymer in DMSO solvent (Figure 5). Similar results have been reported by other researchers in case hyperbranched poly(sulfone amine) and polyether. ${ }^{16,17}$ The decrease of intensity with increase of concentration of the polymer may be attributed by the quenching effect of amino groups present in the structure. Further this decrement via the self-association behaviors of polymers would change with concentration and hence the fluorescence behavior would also change with the same.

\section{Effect of $\mathbf{p H}$}

Hyperbranched polyamine also exhibits $\mathrm{pH}$ dependent fluorescence and shows an increase of fluorescence intensity with the increase of $\mathrm{pH}$ (Figure 6). From this figure, it has been found that with the addition of alkali $(\mathrm{pH}$ 10.6) the intensity of main peak $(455 \mathrm{~nm})$ decreases and the shoulder peak at $390 \mathrm{~nm}$ increases. However, the addition of acid ( $\mathrm{pH} 4$.1-2.8) merges these two peaks and turns to a broad single peak in the fluorescence spectra. These are due to the following facts. It is well known that concentration of acid or base always has some influences on the polarity, hydrogen bonding ability and

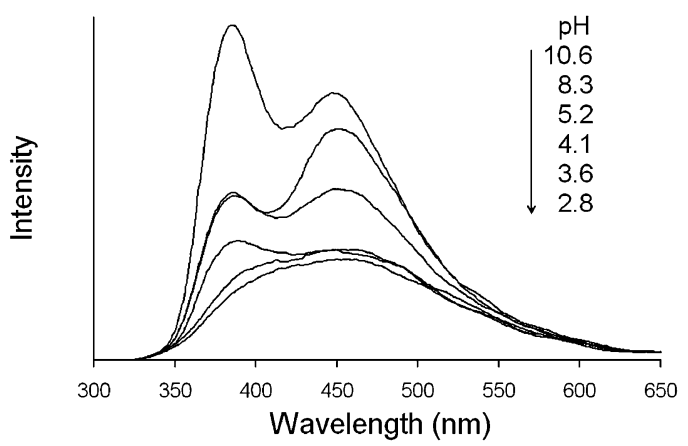

Figure 6. The fluorescence spectra of HBPA with variation of $\mathrm{pH}$. 
solubility and hence on association or aggregation state of the polymer. ${ }^{33,34}$ As this hyperbranched polyamine exhibits all three types of nitrogen atoms in its structure and they (except the tertiary nitrogen of terminal $s$-triazine unit containing two chlorine atoms, as the basicity of the nitrogen atoms is low but number of such moiety is also less as indicated by elemental chlorine percentage, 2.5\%) are protonated under acidic condition, i.e., at low $\mathrm{pH}$, so it is quite obvious that protonation efficiency definitely influenced the fluorescence behavior of the polymer. The increase of fluorescence intensity with the increase of $\mathrm{pH}$ by the addition of dilute alkali $(\mathrm{NaOH})$ and decrease of fluorescence intensity with decreasing $\mathrm{pH}$ by addition of dilute acid $(\mathrm{HCl})$ respectively may be attributed by at least two factors. The first one is reduction of quenching effect by amino group with decreasing $\mathrm{pH}$, i.e., by addition of $\mathrm{HCl}$ solution which makes the polymer as polyelectrolyte. ${ }^{35}$ The hydrogen bonds between different bonded molecules at least partly broken as $\mathrm{pH}$ decreases resulting steady decrease of association domain, whereas in alkaline $\mathrm{pH}$, the amino groups remain free and increase the hydrophobic association of the polymer, which may be due to formation of hydrogen bonding. But the situation at very high $\mathrm{pH}$ (10.6), the primary amino groups may be converted to anionic state though other amino groups may remain as neutral.

\section{CONCLUSIONS}

From this study, it can be concluded that hyperbranched aromatic polyamine with $s$-triazine unit was synthesized and well characterized by using different spectroscopic and analytical techniques. The study shows that the fluorescence of the polymer influenced by variation of concentration, $\mathrm{pH}$ of the medium, dye end capping and the presence of $\mathrm{Cu}^{2+}$ ion. The polymer is also highly thermostable and self-extinguishing. Thus, this hyperbranched polymer may find applications in compact laser devices, light harvesting/light emitting devices, $\mathrm{Cu}$ ion and $\mathrm{pH}$ sensor and so forth.

Acknowledgment. One of the authors, S. S. Mahapatra also expresses his gratitude to CSIR, New Delhi for SRF assistantship through the Grant No. [09/796/(0010)/2007/EMR-I].

Received: May 15, 2008 Accepted: September 12, 2008 Published: November 6, 2008

\section{REFERENCES}

1. C. Gao and D. Yan, Prog. Polym. Sci., 29, 183 (2004).

2. B. Voit, J. Polym. Sci., Part A: Polym. Chem., 38, 2505 (2000).

3. M. Jikei, R. Mori, S. Kawauchi, M. Kakimoto, and Y. Taniguchi,
Polym. J., 34, 550 (2002).

4. H. Chen and J. Yin, J. Polym. Sci., Part A: Polym. Chem., 40, 3804 (2002).

5. N. Spetseris, R. E. Ward, and T. Y. Mayer, Macromolecules, 31, 3158 (1998).

6. J. H. Oh, J. Tang, and S.-H. Lee, Polymer, 42, 8339 (2001).

7. Y. Lim, S. M. Kim, Y. Lee, W. Lee, T. Wang, M. Lee, H. Suh, and J. Park, J. Am. Chem. Soc., 123, 2460 (2001).

8. A. P. de Silva, H. Q. N. Gunaratne, T. Gunnlaugsson, A. J. M. Huxley, C. P. McCoy, J. T. Rademacher, and T. E. Rice, Chem. Rev., 97, 1515 (1997).

9. M. Sukamoto, A. Ueno, and H. Mihra, J. Chem. Soc., Chem. Commun., 1741 (2000).

10. K. Itarni, D. Yamazaki, and I. Yoshida, J. Am. Chem. Soc., 126, 15396 (2004).

11. T. Yasuda, T. Imase, Y. Nakamura, and T. Yamamoto, Macromolecules, 38, 4687 (2005).

12. M. Kucharskiand and W. Mazurkiewicz, Polymer, 23, 1688 (1982).

13. J. Borah, S. S. Mahapatra, D. Saikia, and N. Karak, Polym. Degrd. Stab., 91, 2911 (2006).

14. J. T. Thurston, J. R. Dudley, D. W. Kaiser, I. Hechenbleikner, F. C. Schaefer, and D. Holm-Hansen, J. Am. Chem. Soc., 73, 2981 (1951).

15. S. S. Mahapatra and N. Karak, J. Appl. Polym. Sci., 106, 95 (2007).

16. C. Gao, J. Hou, D. Yan, and Z. Wang, React. Funct. Polym., 58, 65 (2004).

17. C. Gao, D. Yan, B. Zhang, and W. Chen, Langmuir, 18, 3708 (2002).

18. R. Xu, H. Liu, and W. Shi, J. Polym. Sci., Part B: Polym. Phys., 40, 3804 (2002).

19. D. Wu, Y. Lin, C. He, and S. H. Goh, Macromolecules, 38, 9906 (2005).

20. R. Kannan, G.-S. He, T. C. Lin, P. N. Prasad, R. A. Vaia, and L.-S. Tan, Chem. Mater., 16, 185 (2004).

21. J. Brunner and R. Kraemer, J. Am. Chem. Soc., 126, 13626 (2004).

22. R. Krämer, Angew. Chem., Int. Ed., 37, 772 (1998).

23. Q. X. Gao, Y. Xie, X. J. Wang, S. Y. Zhang, T. Hou, and S. C. Lu, Chem. Commun., 26 (2004).

24. R. M. Silverstein, G. C. Bassler, and T. C. Morril, "Spectromtetric Identification of Organic Compounds," 6th ed., Wiley, New York, 1998.

25. K. Takagi, T. Hattori, H. Kunisada, and Y. Yuki, J. Polym. Sci., Part A: Polym. Chem., 38, 4385 (2000).

26. H. E. Birkett, J. C. Cherryman, A. M. Chippendale, J. S. O. Evans, R. K. Harris, M. James, I. J. King, and G. J. McPherson, Magn. Reson. Chem., 41, 324 (2003).

27. C. J. Hawker, R. Lee, and J. M. J. Frechet, J. Am. Chem. Soc., 113 4583 (1991).

28. D. Hölter, A. Burgath, and H. Fery, Acta Polym., 48, 30 (1997).

29. B. D. Sarwade, P. P. Wadgaonkar, and S. S. Mahajan, Eur. Polym. J., 24, 1057 (1988).

30. S. S. Mahapatra and N. Karak, Polym. Degrd. Stab., 92, 947 (2007).

31. D. Braun, R. Ghahary, and T. Ziser, Die Angew. Makromol. Chem., 233, 121 (1995).

32. Z. Liang, Z. Liu, L. Jiang, and Y. Gao, Tetrahedron Lett., 48, 629 (2007).

33. J. Chen, M. Jiang, Y. Zhang, and H. Zhou, Macromolecules, 32, 4861 (1999).

34. Y. Hu, G. L. Smith, M. F. Richardson, and C. L. McCormick, Macromolecules, 30, 3526 (1997).

35. C. Wang, B. Ren, Z. Tong, F. Zeng, X. Lui, S. Wu, and P. Liu, Eur. Polym. J., 41, 185 (2005). 